\title{
Epidemiological Survey of Non-specific Encephalomyelitis in the Chugoku-District
}

\author{
Ziro URAKI \\ Department of Internal Medicine, \\ Hiroshima University School of Medicine
}

A survey was made on the incidence of non-specific encephalomyelitis in the Chugoku-district, and the following results were obtained.

1. Transverse myelitis, disseminated encephalomyelitis and optic neuroencephalomyelitis associated with diarrhea or abdominal pain as initial symptoms were found to occur, infrequently and sporadically, in the Chugoku-district; thirty-five cases were reported in the past six years, most of them being observed in the Sanyodistrict (Hiroshima and Okayama Prefectures).

2. No outbreaks in a family or in a certain locality were encountered.

3. Incidence is increasing in the recent years; six cases in 1959, four cases in 1960, four cases in 1961, seven cases in 1962, and twelve cases in 1963.

4. The disease occurred more often in the summer, and gradually subsided in the fall and winter.

5. Females were significantly more susceptible to this disease than males.

6. Individuals of all ages were susceptible, the least incidence occurring in the ages below twenty years. 